\title{
A Phase I Drug Discovery and One-, Two-, and Three-Class of Indoline-2,3-dione Testing for Anticancer and Cytotoxic Activity
}

\author{
Rahul Hajare* \\ Department of Health Research, Ministry of Health and Family Welfare, India
}

Submission: March 16, 2018; Published: April 04, 2018

*Corresponding author: Rahul Hajare, Department Indian Council of Medical Research, Department of Health Research, Ministry of Health and Family Welfare, New Delhi, India, Tel: 9921707584; Email: rahulhajare@rediffmail.com

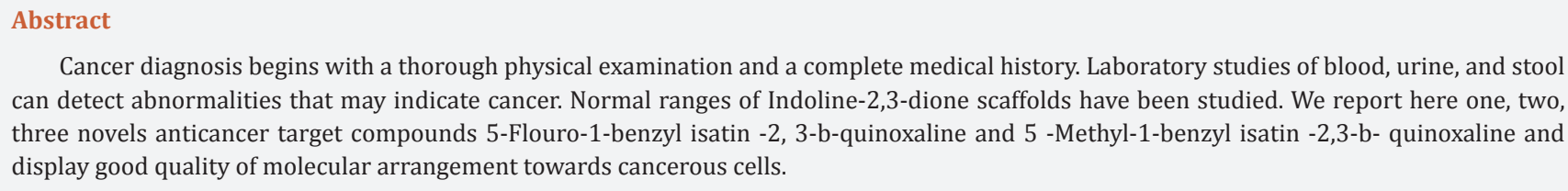

Cancer diagnosis begins with a thorough physical examination and a complete medical history. Laboratory studies of blood, urine, and stool can detect abnormalities that may indicate cancer. Normal ranges of Indoline-2,3-dione scaffolds have been studied. We report here one, two, three novels anticancer target compounds 5-Flouro-1-benzyl isatin -2,3-b-quinoxaline and 5 -Methyl-1-benzyl isatin -2,3-b- quinoxaline and display good quality of molecular arrangement towards cancerous cells.

\section{Introduction}

Cancer has a variety of forms this definition still holds secrete even today and selection removes variation. Cancer comes homologous proteins can differ in their specificities, specific activities, level of expression or some other basic property. However homologous will have some residual similarity in their form and function. Cancer is a biological event has brought some useful innovations in practice to the study of treatment. It was felt there should not be separate drug discovery standard for reduce the dose frequency and reduce adverse effect and shorten time treatment. The natural history of drug discovery could illustrate the complexity in selecting a baseline. Cancer drug discovery is a relatively slow growing chemical agent with an incubation period said to range from a few year to a life time. If any other anatomical symptoms will see during the laboratory trial, it must be treated thoroughly. Indoline-2,3-dione scaffolds is multifunctional expressed by drug discovery series of novel 97 compounds were synthesized for anticancer and cytotoxic activity against different 60 cell line cultures [1]. 07 compounds were selected on molecular recognition and screened for anticancer study [2]. Among those is screened compounds RH8, RH 42, RH84, RH89, RH90, RH 94, RH97 found good quality of anticancer activity against most of the cancer cell line with ranges from -77.23 to $55.85 \%$ growth. Target compound 5-Flouro-1-benzyl isatin -2, 3-b-quinoxaline (RH-89) display -77.23 to $7.31 \%$ growth \& another target compound RH 94 display -64.78 to $65.14 \%$ growth against most of the leukemic cell lines, while RH-08 has shown very good activity against CCRF-CEM cell line with $-31.85 \%$ growths and weak anticancer activity against breast cancer cell line with $53.47 \%$ growth, but failed to respond against other leukemic cell lines. RH-89 has also shown moderate activity against nonsmall cell-lung cancer (HOP-62) with 34.69\% growth. RH-90 has shown good anticancer activity against leukemic cell line SR with $18.70 \%$ growth and weak activity against Non small cell lung cancer HOP-92 and NCL-H322M with 57.14 and 57.20\% growth respectively. The cytotoxic property [3] of Indoline-2,3-dione scaffolds, among 3-benzohydrazide (RH 06-64) series, RH-22 and 28 were found to be most cytotoxic against L-1210 cell line ( 9 and $6 \mu \mathrm{g} / \mathrm{ml}$ ). RH-33 was found to be cytotoxic against CEM/0 cell line $(6.9 \mu \mathrm{g} / \mathrm{ml})$. Among 3-isonicotinohydrazide derivatives (RH 65-72), RH-70 was found to have better cytotoxic property against all three types of cell lines $(6.1-6.7 \mu \mathrm{g} / \mathrm{ml})$. From 3-quinazolin-4(3H)-one series ( $\mathrm{RH} 74-82), \mathrm{RH}-75$ was found to be better cytotoxic against Molt4/8 cell line $(6.0 \mu \mathrm{g} / \mathrm{ml})$ and RH-77 was found to have better cytotoxicity against L1210 cell line $(9 \mu \mathrm{g} / \mathrm{ml})$ remaining compounds $\mathrm{RH}-79,80,82$ have shown moderate to weak cytotoxic property. Among 6H-indolo [2,3-b] quinoxaline derivatives (RH 83-96), RH-92 have shown better cytotoxic property against $\mathrm{L} 1210$ cell line $(7.2 \mu \mathrm{g} / \mathrm{ml})$.

\section{Compounds and Reagents}

All chemicals used were procured from Sigma-Aldrich. The purity of chemicals was checked before use and purified. Melting points were recorded by open capillary tube method and uncorrected. Purity of all the synthesized compounds was 
assessed by thin layer chromatography on silica Gel-G'(100-200 mesh) plates. Structures of compounds were confirmed by FTIR, ${ }^{1} \mathrm{HNMR}$ and Mass spectra. IR spectra were recorded in $\mathrm{KBr}$ disk on Nicolate IR-Instrument and reported in $\mathrm{cm}^{-1}$. Mass spectra were recorded on Shimadzu-LCMS 2010 EV-single quadrapole instrument. ${ }^{1} \mathrm{HNMR}$ spectra were recorded on the BRUKER-300 instrument with TMS as the internal standard in $\mathrm{CDCl}_{3} / \mathrm{DMSO}$ as a solvent. Total 97 derivatives of Indoline-2,3-dione scaffolds have been synthesized by Sandmeyer process methodology using N-methyl morpholine, substituted benzylchlorides, isoniazid, substituted benzoylhydrazide, quinazoline, orthophenylenediamine, halogens and alkyl groups as substituent and compounds were recrystallized by using ethanol-chloroform mixture. Synthesized compounds and melphalan as a standard were also evaluated for their in vitro cytostatic activity against human Molt 4/C8 and CEM T-lymphocytes as well as murine leukemia L1210 and anticancer activity against Leukemia, Non-Small Cell-Lung Cancer, Colon Cancer, and Breast Cancer. Synthesized compounds were screened for anticancer activity at National Cancer Institute, Maryland, USA and Rega Institute, Belgium respectively (Figure 1).

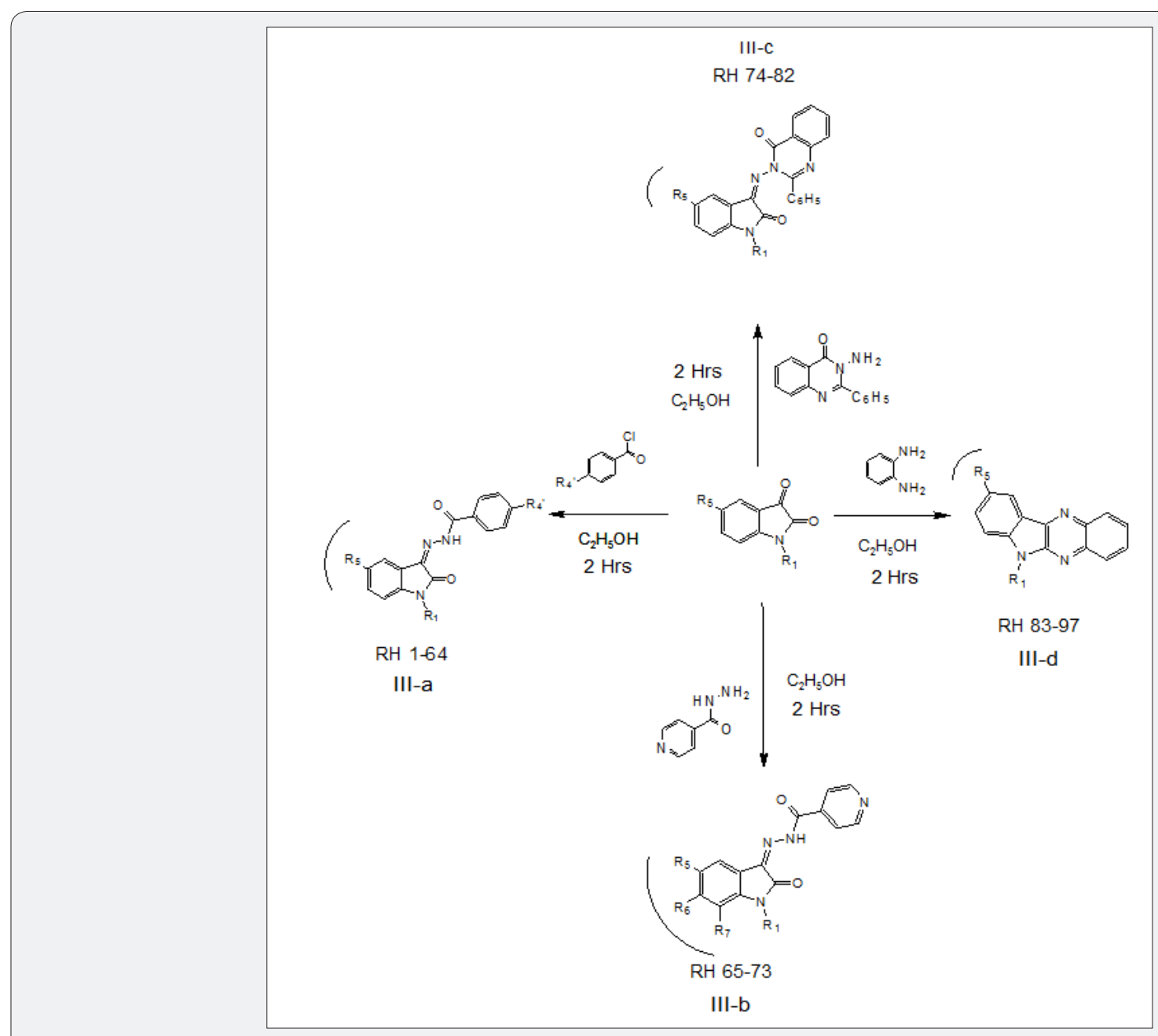

Figure 1: Phase I Drug Discovery.

$\mathrm{R}_{1}=\mathrm{H},-\mathrm{CH}_{3}, \mathrm{C}_{6} \mathrm{H}_{5}-\mathrm{CH}_{2}-, 4^{\prime}-\mathrm{CL}-\mathrm{C}_{6} \mathrm{H}_{4}-\mathrm{CH}_{2}-, 4^{\prime}-\mathrm{F}_{-}-\mathrm{C}_{6} \mathrm{H}_{4}-\mathrm{CH}_{2}-, 4^{\prime}-\mathrm{CH}_{3}-\mathrm{C}_{6} \mathrm{H}_{4}-\mathrm{CH}_{2}-,-\mathrm{CH}_{2}-\mathrm{N}_{-} \mathrm{C}_{4} \mathrm{H}_{8} \mathrm{O}, \mathrm{R}_{4}^{\prime}=\mathrm{H}, \mathrm{Cl}, \mathrm{Br}, 3,4,5$ trimethoxy, $\mathrm{NO}_{2}, \mathrm{NH}_{2}, \mathrm{R}_{5}=\mathrm{H}, \mathrm{Cl}, \mathrm{F}, \mathrm{Br},-\mathrm{CH}_{3}, \mathrm{R}_{6}=\mathrm{CL}, \mathrm{R}_{7}=\mathrm{Br}$

\section{Result and Discussion (Tables 1 \& 2).}

\section{Chemistry}

RH8; MF C15H10N302Cl, Yellowish fluffy mass, MW 299.71, MP 280-82,\% Yield 49, IR 3226 (-NH), 3044 (-CH, ar.), 1701 (>CO), 1678 (>CO). NMR 14.03 (d, 1H, NH.), 11.28 (s, 1H, NH), 7.88-7.84 (m 2H, ar.), 7.59-7.42 (m, 4H, ar.), 7.25-7.23 (m, $1 \mathrm{H}$, ar.), 6.87-6.84 (m, 1H, ar.). m/s 299 (299.29)
RH 42; $\mathrm{MF} \mathrm{C}_{22} \mathrm{H}_{16} \mathrm{~N}_{30} 2_{\text {Cl}}$, Yellowish-fibrous mass, MW 389.83, MP 170-72, \% Yield 81, IR 3363 (-NH), 3196 (-CH, ar.), 3028 (CH, alip.), 1750 (>CO), 1682 (>CO). NMR 14.04 (s, 1H, NH.), 7.97(d, $2 \mathrm{H}$, ar.), 7.82 (s, $1 \mathrm{H}$, ar.), 7.46 (t, $2 \mathrm{H}$, ar.), 7.48 (t, $1 \mathrm{H}, \mathrm{ar}$ ), 7.257.04 (m, 5H, ar.), 6.76 (d, 1H, ar.), 4.87 (s, $2 \mathrm{H}, \mathrm{CH}_{2}$ ), 2.25 (s, 3H, $\mathrm{CH}_{3}$ ). m/s 389 (389.42)

RH84: $M F \mathrm{C}_{21} \mathrm{H}_{14} \mathrm{FN}_{3}$, Reddish Fluffy Mass, MW 327.35,MP 162-13,\% Yield 72,IR 3045-3013 (-CH, ar.), 2954-2849 (-CH, 


\section{Organic \& Medicinal Chemistry International Journal}

alip.), 1610, (>C=C), 1580 (>C=N).,NMR 8.51 (d, 1H, ar.), 8.348.32 (m, $1 \mathrm{H}, \mathrm{ar}.), 8.16$ (d, 1H, ar.), 7.80-7.76 (m, 1H, ar.), 7.737.69 (m, 1H, ar.), 7.63 (t, 1H, ar.), 7.41-7.31(m, 4H, ar.), $7.00(\mathrm{t}$, $2 \mathrm{H}$, ar.), 5.69 (s, $2 \mathrm{H},-\mathrm{CH}_{2}$ ).,

RH89, MF $\mathrm{C}_{21} \mathrm{H}_{14} \mathrm{FN}_{3}$, Orange Mass, MW 327.35,MP 220-22,\% Yield 68, IR 3066-3004 (CH, ar.), 2904-2849 (CH, alip.), 1618 $(>\mathrm{C}=\mathrm{C}), 1582(>\mathrm{C}=\mathrm{N})$., NMR $8.38(\mathrm{~d}, 1 \mathrm{H}, \mathrm{ar}), 8.24-8.19(\mathrm{~m}, 2 \mathrm{H}$, ar.), 7.86-7.82 (m, 1H, ar.), 7.79-7.74 (m, 1H, ar.), 7.42 (d, 1H, ar.), 7.39-7.32 (m, 2H, ar.), 7.31-7.28 (m, 4H, ar.), 5.77 (s, 2H, $-\mathrm{CH}_{2}$ ).

RH90; $\mathrm{MF} \mathrm{C}_{22} \mathrm{H}_{16} \mathrm{FN}_{3}$, Yellowish Mass, MW 341.38,MP 17780,\% Yield 71,IR 3054-3029 (CH, ar.), 2917-2849 (-CH, alip.), $1613(>\mathrm{C}=\mathrm{C}), 1585$ (>C=N). NMR 8.33 (d, 1H, ar.), 8.18-8.15 (m, $1 \mathrm{H}$, ar.), 7.81-7.77 (m, 1H, ar.), 7.73-7.69 (m,1H, ar.), 7.37-7.29 (m, 3H, ar.), 7.22 (d, 2H, ar.), 7.12 (d, 2H, ar.), 5.68 (s, $2 \mathrm{H},-\mathrm{CH}_{2}$ ), $2.30\left(\mathrm{~s}, 3 \mathrm{H},-\mathrm{CH}_{3}\right)$.

RH 94; $M F C_{22} \mathrm{H}_{17} \mathrm{~N}_{3}$, Reddish Fluffy Mass, MW 323.39, MP 215-18, \% Yield 75, IR 3059-3024 (CH, ar.), 2966-2850 (-CHalip.), 1616 (>C=C), $1578(>C=N)$.NMR 8.38-8.36 (m, 2H, ar.), 8.20-8.18 (m, 1H, ar.), 7.83-7.79 (m, 1H, ar.), 7.76-7.72 (m, $1 \mathrm{H}$, ar.), 7.49 (d, $1 \mathrm{H}$, ar.), $7.38-7.29$ (m, 6H, ar.), $5.67\left(\mathrm{~s}, 2 \mathrm{H},-\mathrm{CH}_{2}\right.$ ), $2.59\left(\mathrm{~s}, 3 \mathrm{H},-\mathrm{CH}_{3}\right)$.

Table 1: Anti cancer and cytotoxicity result.

\begin{tabular}{|c|c|c|c|c|c|c|c|c|}
\hline Sl. No. & $\begin{array}{l}\text { Panel/ Cell } \\
\text { Line }\end{array}$ & $\begin{array}{c}\text { Percentage } \\
\text { of Growth } \\
(\%)\end{array}$ & & & & & & \\
\hline & & RH-08 & RH-42 & RH-84 & RH-89 & RH-90 & RH-94 & RH-97 \\
\hline \multicolumn{9}{|c|}{ 1.0 Leukemia } \\
\hline 1.1 & CCRF-CEM & -31.85 & 10.05 & 99.92 & -77.23 & 70.99 & -64.78 & 77.05 \\
\hline 1.2 & HL-60(TB) & 65.13 & -- & 88.21 & 88.47 & 30.11 & 65.14 & 75.21 \\
\hline 1.3 & K-562 & 97.17 & 91.86 & 83.91 & 76.50 & 69.85 & 32.40 & 65.18 \\
\hline 1.4 & MOLT-4 & 91.22 & 80.31 & 84.36 & 7.31 & 75.09 & 45.51 & 91.04 \\
\hline 1.5 & RPMI-8226 & 94.45 & 77.82 & 90.92 & 50.03 & 70.99 & 58.90 & 57.91 \\
\hline 1.6 & SR & 96.92 & 89.34 & 107.13 & -65.87 & 18.70 & -2.81 & 103.69 \\
\hline \multicolumn{9}{|c|}{ 2.0 Non-Small Cell-Lung Cancer } \\
\hline 2.1 & A549/ATCC & 104.69 & 83.25 & 97.89 & 97.22 & 81.56 & 94.32 & 96.53 \\
\hline 2.2 & EKVX & 93.51 & 81.29 & 98.96 & 109.76 & 90.39 & 96.33 & 102.89 \\
\hline 2.3 & HOP-62 & 99.72 & 93.16 & 97.35 & 34.69 & 85.09 & 47.75 & 106.14 \\
\hline 2.4 & HOP-92 & 80.99 & 94.76 & 88.55 & 95.14 & 57.14 & 101.91 & 44.90 \\
\hline 2.5 & NCL-H-226 & 99.96 & 80.53 & 110.86 & 86.06 & 97.72 & 86.76 & 102.22 \\
\hline 2.6 & NCL-H-23 & 92.99 & 79.46 & 88.12 & 82.36 & 90.30 & 96.03 & 88.75 \\
\hline 2.7 & NCL-H 322M & 115.93 & 76.95 & 98.74 & 88.86 & 57.20 & 96.64 & 97.96 \\
\hline 2.8 & NCL-H-460 & 101.04 & 48.01 & 97.74 & 79.63 & 79.39 & 95.29 & 96.11 \\
\hline 2.9 & NCL-H-522 & 102.93 & 85.25 & 88.20 & 97.22 & 72.35 & 94.32 & 72.89 \\
\hline \multicolumn{9}{|c|}{ 9.0 Breast Cancer } \\
\hline 4.1 & MCF-7 & 53.47 & 80.66 & 80.48 & 83.53 & 80.20 & 103.26 & 84.45 \\
\hline
\end{tabular}

RH97: $\mathrm{MF} \mathrm{C}_{21} \mathrm{H}_{15} \mathrm{FN}_{3}$, Yellowish Mass, MW 309.36,MP 173-75,\% Yield 60 IR 3048-3030 (CH, ar.), 2908-2856 (-CH, alip.),1610 (>C=C), 1562(>C=N).NMR 8.32 (d, 2H, ar.), 8.14 (d, $1 \mathrm{H}$, ar.) 7.79-7.75 (m, 1H, ar.), 7.71-7.68 (m, 1H, ar.), 7.45 (d, $1 \mathrm{H}$, ar.), 7.32-7.23 (m, $3 \mathrm{H}$, ar.) 6.98-6.95 (m, 2H, ar.), 5.66, (s, $2 \mathrm{H}$, $\left.-\mathrm{CH}_{2}\right), 2.55\left(\mathrm{~s}, 3 \mathrm{H}, \mathrm{CH}_{3}\right)$.

RH-22; $\mathrm{MF} \mathrm{C}_{15} \mathrm{H}_{9} \mathrm{BrFN}_{3} \mathrm{O}_{2}$, Orange gritty powder, MW 362.15, MP >310, \% Yield 57, IR 3229 (-NH), 3056 (-CH, ar.), 1681 (>CO), $1620(>\mathrm{C}=\mathrm{C}), 1552(\mathrm{C}=\mathrm{N}), 1463(\mathrm{C}-\mathrm{H}) . \mathrm{NMR}$

RH28; Yellowish fluffy mass, MW 423.06, MP >310, \% Yield 84,IR 3263 (-NH), 3035 (-CH, ar.), 2783 (-CH, alip), 1672 (>CO), $1665(>\mathrm{C}=\mathrm{C}), 1529$ (C=N), $1467(\mathrm{C}-\mathrm{H})$.

RH65; $\mathrm{MF} \mathrm{C}_{21} \mathrm{H}_{8} \mathrm{~N}_{4} \mathrm{O}_{2} \mathrm{Cl}$, Yellowish fluffy mass, MW 390.82, MP 197-99, \% Yield 76 IR3252 (-NH), 3073 (-CH, ar.), 2918, (CH, alip.), 2850 (CH, alip.) 1691 (>CO). 13.87 (s, 1H, -NHCO), 8.873 (d, 2H, ar.) 7.813 (d, 2H, ar.), 7.765 (s, 1H, ar.), 7.611 (d, 1H, ar.), 7.339 (m, 4H, ar.), 7.05 (d, 1H, ar.), 5.018 (s, 2H,- $-\mathrm{CH}_{2}$ )

$\mathrm{RH} 72 ; \mathrm{MF} \mathrm{C}_{14} \mathrm{H}_{2} \mathrm{~N}_{4} \mathrm{O}_{2} \mathrm{ClF}$, Orange gritty powder, MW 318.69, MP299-300, \% Yield 70, IR 3249 (-NH), 3070 (-CH, ar.), 29312849 (-CH, alip.), 1700-1691 (>CO), $1613(>\mathrm{C}=\mathrm{C}), 1520(\mathrm{C}=\mathrm{N})$, 1472 (C H). NMR13.95 (s, 1H, - NHCO), 8.86 (d, 2H, ar.), 7.80 (d, 2H, ar.), 7.5 (s, 1H, ar.), 7.305 (m, 6H, ar.), 6.9685 (d, 1H, ar.), $4.987\left(\mathrm{~s}, 2 \mathrm{H},-\mathrm{CH}_{2}-\right.$ ), $2.296\left(\mathrm{~s}, 3 \mathrm{H},-\mathrm{CH}_{3}\right)$. 


\section{Organic \& Medicinal Chemistry International Journal}

\begin{tabular}{|c|c|c|c|c|c|c|c|c|}
\hline 4.2 & $\begin{array}{c}\text { MDA- } \\
\text { MB-231/ } \\
\text { ATCC }\end{array}$ & 105.18 & 88.38 & 111.89 & 71.11 & 72.60 & 81.36 & 95.92 \\
\hline 4.3 & HS 578T & 119.56 & 85.40 & 104.26 & 97.08 & 68.94 & 110.37 & 94.26 \\
\hline 4.4 & BT-549 & 115.46 & 90.84 & 109.74 & 113.24 & 103.21 & 96.06 & 103.89 \\
\hline 4.5 & T-47D & 81.47 & 71.04 & 81.38 & 125.52 & 88.018 & 153.29 & 89.51 \\
\hline 4.6 & MDA-MB- 468 & 81.99 & 92.64 & -- & -- & -- & -- & -- \\
\hline
\end{tabular}

Table 2: Inhibitory effect on cell proliferation

\begin{tabular}{|c|c|c|c|c|c|c|c|}
\hline Compound & $\begin{array}{c}\text { Types of Cell } \\
\text { Line }\end{array}$ & Compound & $\begin{array}{c}\text { Types of Cell } \\
\text { Line }\end{array}$ & & & & \\
\hline & L1210 & Molt4/8 & CEM/0 & & L1210 & Molt4/8 & CEM/0 \\
\hline RH-6 & $12 \pm 3$ & $36 \pm 12$ & $91 \pm 20$ & RH-83 & $38 \pm 24$ & $23 \pm 10$ & $38 \pm 6$ \\
\hline RH-22 & $9 \pm 1$ & $23 \pm 6$ & $33 \pm 10$ & RH-84 & $199 \pm 27$ & $170 \pm 18$ & $188 \pm 40$ \\
\hline RH-25 & $16 \pm 3$ & $55 \pm 9$ & $23 \pm 2$ & RH-87 & $31 \pm 1$ & $>200$ & $\geq 200$ \\
\hline RH-26 & $5 \pm 0.9$ & $69 \pm 15$ & $15 \pm 9$ & RH-88 & $\geq 200$ & $23 \pm 8$ & $>200$ \\
\hline RH-28 & $6.5 \pm 12$ & $15 \pm 7$ & $125 \pm 25$ & RH-89 & $30 \pm 15$ & $>200$ & $62 \pm 17$ \\
\hline RH-31 & $>500$ & $12 \pm 3$ & $46 \pm 8$ & RH-91 & $100 \pm 5$ & $132 \pm 35$ & $78 \pm 49$ \\
\hline RH-33 & $126 \pm 16$ & $27 \pm 8$ & $12 \pm 4$ & RH-92 & $7.2 \pm 5.3$ & $222 \pm 25$ & $247 \pm 28$ \\
\hline RH-70 & $6.7 \pm 3.6$ & $6.6 \pm 0.6$ & $6.1 \pm 0.0$ & RH-93 & $50 \pm 27$ & $>500$ & $400 \pm 142$ \\
\hline RH-75 & $36 \pm 6.6$ & $6 \pm 0.9$ & $26 \pm 1.1$ & RH-94 & $84 \pm 24$ & $126 \pm 52$ & $94 \pm 7$ \\
\hline RH-79 & $68 \pm 14$ & $19 \pm 7$ & $17 \pm 8$ & RH-95 & $84 \pm 8$ & $92 \pm 8$ & $83 \pm 20$ \\
\hline RH-80 & $15 \pm 1.1$ & $123 \pm 32$ & $91 \pm 12$ & RH-96 & $32 \pm 16$ & $424 \pm 81$ & $>500$ \\
\hline RH-82 & $12 \pm 2$ & $12 \pm 3$ & $32 \pm 11$ & Melphalan & $2.1 \pm 0.02$ & $3.2 \pm 0.6$ & $2.5 \pm 0.2$ \\
\hline
\end{tabular}

*50\% inhibitory concentration $\left(\mathrm{IC}_{50}\right)$ value, expressed in uM

\section{Conclusion}

A comparison of the synthesized and evaluated novel Indoline-2,3-dione scaffolds and its series which showed nanomolar activity against most of the leukemia cell line and other cancers cell line. Taste compounds RH8, RH 42, RH84, RH89, RH90, RH 94, and RH97 possess inhibitory activity. Target compound RH89 and RH 94 displays -77.23 to $7.31 \%$ and another target compound - $64.78 \%$ to $65.14 \%$ growth respectively. All the 97 compounds designed analogs with chemical substitutions at the various position confirmed by analytical model showing better interactive scores respectively. Molecular recognition studies in the series removal of alky group from 4'-position of N-benzyl ring causes loss in activity (RH-71) and replacement of benzyl group by $-\mathrm{H}$ makes the compound inactive (RH-72), while RH-08 has shown very good activity against CCRF-CEM cell line with $-31.85 \%$ growths and weak anticancer activity against breast cancer cell line with $53.47 \%$ growth, but failed to respond against other leukemic cell lines. RH-89 has also shown moderate activity against non-small celllung cancer (HOP-62) with $34.69 \%$ growth. RH-90 has shown good anticancer activity against leukemic cell line SR with $18.70 \%$ growth and weak activity against Non small cell lung cancer HOP-92 and NCL-H322M with 57.14 and 57.20\% growth respectively. The comparison of activity with structure similarity and differences in substitution reveals that compounds with quinoxaline moiety are more active than benzohydrazide moiety (RH-89 \& 94> RH-08). Further, 5-fluoro or 5-methyl substituted compounds (RH-89 \& 94) were more active than un-substituted analogues (RH-97). It was also observed that any substitution on the 4' position of N-benzyl ring causes loss in activity (RH-90 \& 97). Similarly among benzohydrazide series compounds with chlorine substitution at $5^{\text {th }}$ position is active (RH-08), however $\mathrm{N}$-benzylation reduces the activity (RH-42). However role of $\mathrm{N}$-benzyl group has mixed effect on the activity. The cytotoxic results of benzohydrazide series show that RH-22 and RH-28 were most cytotoxic against $\mathrm{L}-1210$ cell line $(9$ and $6 \mu \mathrm{g} / \mathrm{ml})$. RH33 was found to be cytotoxic against CEM/0 cell line $(6.9 \mu \mathrm{g} / \mathrm{ml})$. The activity report shows that presence of halogen increases the cytotoxic property. Replacement of halogen from 4' position of benzohydrazide causes loss in activity as in case of RH-06, 25, 26, 28 and 31. Among 3-isonicotinohydrazide derivatives RH-70 was found to have better cytotoxic property against all three types of cell lines $(6.1-6.7 \mu \mathrm{g} / \mathrm{ml})$. Removal of alky group from 4'-position of N-benzyl ring causes loss in activity (RH-71) and replacement of benzyl group by $-\mathrm{H}$ makes the compound inactive (RH-72). It shows that halogen at $5^{\text {th }}$ position and alkyl group at $4^{\prime}$ position of N-benzyl ring is essential for activity. From 3-quinazolin$4(3 \mathrm{H})$-one series RH-75 was found to be better cytotoxic against Molt4/8 cell line $(6.0 \mu \mathrm{g} / \mathrm{ml})$ and RH-77 was found to have better cytotoxicity against $\mathrm{L} 1210$ cell line $(9 \mu \mathrm{g} / \mathrm{ml})$. Among $6 \mathrm{H}$-indolo [2,3-b]quinoxaline derivatives RH-92 has shown better cytotoxic 
property against L1210 cell line $(7.2 \mu \mathrm{g} / \mathrm{ml})$ which shows the importance of halogen at $5^{\text {th }}$ position and presence of N-benzyl moiety in the compound. Alteration in the combination leads to loss in activity (RH-84, 87, 88, 89, 90, 91, 93-96). Based on the results without any halogen has either very weak activity or inactive and none of them are close to the standard Malphalan (2.1-3.2 $\mu \mathrm{g} / \mathrm{ml})$ [4-11].

\section{Acknowledgment}

This study has been guided under the guidance of Renowned Laboratory Scientist Respected Dr. Ramesh Paranjape, Founder National AIDS Research Institute India. I express my sincere gratitude towards Respected Sir for motivation and being great knowledge source for this work

\section{References}

1. Rahul Hajare (2017) Response to a 2 Indolinones laboratory based regimen analogous in cancerous-infected cells. Biomed Res Ther 4(S).

2. Hajare R (2017) Can Otolaryngology Capture Window Cancer in Middle Adulthood? Ann Clin Lab Res 5(4): 205.

3. Rahul Hajare (2017) Classical Technology Can Run Away Impurity in Pharmaceuticals Frugal Innovation Lesson from Classic Innovation System. Organic \& Medicinal Chem IJ 2(4):555592.

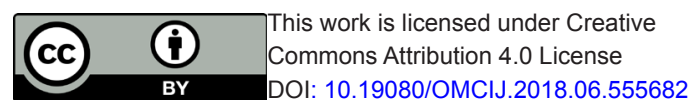

4. Rahul Hajare (2017) Tertiary Care in Impurity Trends New Pattern Discovery. Organic \& Medicinal Chem IJ 2(4): 555591.

5. Rahul Hajare (2017) New pattern clinical study of adverse drug reaction to ART may lead to chase drug discovery in anti-retroviral drug towards zero adverse drug reaction. Curr Res Integr Med 2(2).

6. Rahul Hajare (2017) Characterization of Inconsistent Unspecified Impurity Associated with Specified Impurity and Adjacent to Other Detectable Impurities Who Have Not Listed in Pharmacopoeias in Ciprofloxacin Hydrochloride. Chem Sci J 8(3): 1-3.

7. Hajare R (2018) The Curve Penis May Hold Secret to Cause of Cancer. A Profile of Primary Level Attending Review Treatment. J Nanomed Nanosci pp. 143.

8. Rahul Hajare (2018) There is no Cure for the Cancer of Stupidity. Organic \& Medicinal Chem IJ 5(1): 555655.

9. Rahul Hajare (2017) The Aggression is an Early Cause of Cancer. A Narrative Review of Classical to Modern Scientific Literature. Chronicle of Medicine and Surgery 1(2): 67-68.

10. Rahul Hajare (2017) Response to a 2-Indolinones laboratory based regimen analogous in cancerous-infected cells. The $3^{\text {rd }}$ International Conference Innovations in Cancer Research and Regenerative Medicine p. 10-13.

11.Rahul Hajare (2017) New Technology of Nucleoside AIDS Virus Resistance Drug Key Intermediate Diethyl (Tosyloxy) Methylphosphonate. SF Drug Deliv Res J 1:1.

\begin{tabular}{l} 
Your next submission with Juniper Publishers \\
will reach you the below assets \\
- Quality Editorial service \\
- Swift Peer Review \\
- Reprints availability \\
- E-prints Service \\
- Manuscript Podcast for convenient understanding \\
- Global attainment for your research \\
- Manuscript accessibility in different formats \\
( Pdf, E-pub, Full Text, Audio) \\
- Unceasing customer service \\
Track the below URL for one-step submission \\
https://juniperpublishers.com/online-submission.php \\
\hline
\end{tabular}

